\title{
Quantum enhancement of accuracy and precision in optical interferometry
}

\author{
Florian Kaiser $^{1,2,3}$, Panagiotis Vergyris ${ }^{1}$, Djeylan Aktas ${ }^{1}$, Charles Babin ${ }^{1,4}$, Laurent Labonté ${ }^{1}$ \\ and Sébastien Tanzilli ${ }^{1}$
}

White-light interferometry is one of today's most precise tools for determining the properties of optical materials. Its achievable precision and accuracy are typically limited by systematic errors due to a high number of interdependent data-fitting parameters. Here, we introduce spectrally resolved quantum white-light interferometry as a novel tool for optical property measurements, notably, chromatic dispersion in optical fibres. By exploiting both spectral and photon-number correlations of energy-time entangled photon pairs, the number of fitting parameters is significantly reduced, which eliminates systematic errors and leads to an absolute determination of the material parameter. By comparing the quantum method to state-of-the-art approaches, we demonstrate the quantum advantage of 2.4 times better measurement precision, despite requiring 62 times fewer photons. The improved results are due to conceptual advantages enabled by quantum optics, which are likely to define new standards in experimental methods for characterising optical materials.

Light: Science \& Applications (2018) 7, 17163; doi:10.1038/lsa.2017.163; published online 23 March 2018

Keywords: chromatic dispersion; interferometry; quantum optics; quantum metrology

\section{INTRODUCTION}

Quantum technologies have received substantial attention as a means to improve the resolution and precision of metrological tasks by reducing statistical errors due to quantum noise ${ }^{1-8}$. Far less attention has been given to their ability to reduce systematic errors. However, statistical and systematic errors are of equal importance in any measurement, and the latter are typically more difficult to characterise. Notable examples of quantum-improved measurements are the combination of multiple fundamental electronic quantum effects for a more accurate definition of the ampere ${ }^{9}$ and quantum-correlated 'twin photon beams' in establishing absolute and universal optical power standards ${ }^{10}$. In this letter we demonstrate a new use of quantum optics to reduce systematic errors in the technologically prominent application of spectrally resolved white-light interferometry (WLI). WLI is used for precise measurements of chromatic dispersion, that is, the second derivative of the wavelength-dependent optical phase. Classical WLI, however, requires precise interferometer equalization ${ }^{11,12}$ and is influenced by third-order dispersion ${ }^{13,14}$. This leads to systematic errors that are difficult to account for.

We eliminate these drawbacks by inferring chromatic dispersion using energy-time entangled photon pairs and coincidence counting to measure spectral correlation functions. In addition, we exploit photon-number correlations to achieve a twofold resolution enhancement. Our results demonstrate that this new strategy outperforms the precision and accuracy of previous quantum ${ }^{15,16}$ and stateof-the-art techniques ${ }^{11,12}$. Moreover, because our approach is essentially alignment-free, it enables the use of the same interferometer in a user-friendly manner for analysing a wide variety of different optical materials in terms of type, optical properties, length, etc.

\section{Standard WLI}

The standard scheme for WLI is shown in Figure 1a. The emission of a white-light source is directed to an interferometer in which the reference arm is free-space (with well-known optical properties) and the other arm comprises the sample under test (SUT). Recombining both arms at the output beam splitter leads to an interference pattern for which the intensity follows $I \propto 1+\cos (\phi(\lambda))$, with $\phi(\lambda)=\frac{2 \pi}{\lambda}\left(n(\lambda) L_{\mathrm{s}}-L_{\mathrm{r}}\right)$. Here, $\lambda$ represents the wavelength, $L_{\mathrm{r}}$ and $L_{\mathrm{s}}$ are the physical lengths of the reference arm and the SUT, respectively, and $n(\lambda)$ is the effective refractive index of the SUT. It is worth noting that interference is observed only when the interferometer is precisely balanced to within the larger of the coherence length of the white-light source and the coherence length imposed by the resolution of the spectrometer, which is typically on the order of microns to millimetres ${ }^{11,12}$. In this case, the phase term reads (more

\footnotetext{
${ }^{1}$ Université Côte d'Azur, Institut de Physique de Nice (INPHYNI), Nice 06108, France; ${ }^{2}$ Now at 3. Physikalisches Institut, Universität Stuttgart, Stuttgart 70569, Germany; ${ }^{3}$ Now at Center for Integrated Quantum Science and Technology (IQST), Stuttgart 70569, Germany and École Normale Supérieure de Lyon, Lyon 69364, France

Correspondence: F Kaiser, Email: f.kaiser@physik.uni-stuttgart.de

Received 31 July 2017; revised 10 November 2017; accepted 20 November 2017; accepted article preview online 26 November 2017
} 
a

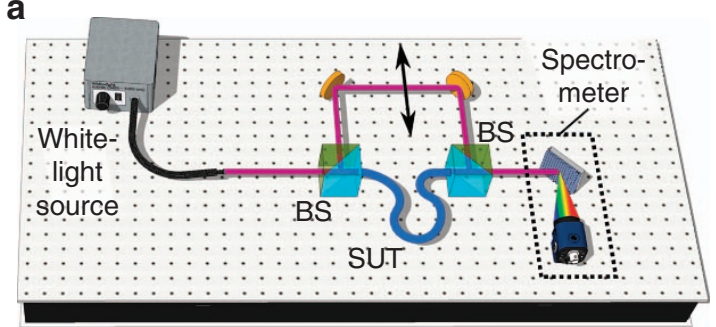

b

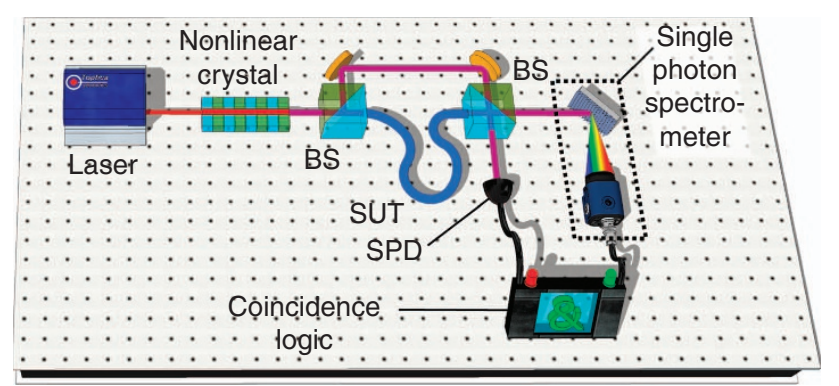

Figure 1 Typical experimental set-ups. (a) Standard spectrally resolved WLI. (b) Quantum WLI. BS, beam splitter.

details are given in the Supplementary Information):

$$
\phi(\lambda) \approx 2 \pi L_{\mathrm{s}}\left(\left.\frac{1 \mathrm{~d}^{2} n}{2 \mathrm{~d} \lambda^{2}}\right|_{\lambda_{0}} \cdot \frac{(\Delta \lambda)^{2}}{\lambda_{0}+\Delta \lambda}+\left.\frac{1 \mathrm{~d}^{3} n}{6 \mathrm{~d} \lambda^{3}}\right|_{\lambda_{0}} \cdot \frac{(\Delta \lambda)^{3}}{\lambda_{0}+\Delta \lambda}\right)+\phi_{\text {off }}
$$

Here, $\lambda_{0}$ represents the stationary phase point, that is, the wavelength at which the absolute phase difference between the two interferometer arms is exactly zero. In standard WLI, $\lambda_{0}$ is extracted experimentally by identifying the symmetry point of the observed interferogram ${ }^{11,12}$. Additionally, $\Delta \lambda=\lambda-\lambda_{0}$, and $\phi_{\text {off }}$ is a constant offset phase. Provided that $L_{\mathrm{S}}$ is precisely known, the optical material parameters $\frac{d^{2} n}{d \lambda^{2}} \mid \lambda_{0}$ and $\frac{\mathrm{d}^{3} n}{\mathrm{~d}^{3}} \mid \lambda_{0}$ can be extracted from a fit to the data as a function of $\Delta \lambda$. It is noteworthy that the three free parameters, that is, $\lambda_{0}, \frac{\mathrm{d}^{2} n}{\mathrm{~d} \lambda^{2}} \mid \lambda_{0}$ and $\frac{\mathrm{d}^{3} n}{\mathrm{~d} \lambda^{3}} \mid \lambda_{0}$, are usually all interdependent in a non-trivial manner such that uncertainties in one parameter systematically induce uncertainties in the others. In fact, the high number of fitting parameters required and the necessity to re-equilibrate the interferometer for every new SUT are the main limiting factors of this technique ${ }^{13,14}$.

However, more accurate optical measurements are eagerly demanded in almost all fields involving optics. A special focus is made on the optical parameter $\left.\frac{\mathrm{d}^{2} n}{\mathrm{~d} \lambda^{2}}\right|_{\lambda_{0}}$, as it is directly related to the chromatic dispersion coefficient $D=-\frac{\lambda_{0}}{c} \cdot \frac{\mathrm{d}^{2} n}{\mathrm{~d}^{2} \mid} \lambda_{0}$, where $c$ is the speed of light ${ }^{13,17-23}$. Chromatic dispersion causes optical pulse broadening, and more accurate measurements of $D$ would have significant repercussions for optimising today's telecommunication networks, developing new-generation pulsed lasers and amplifiers, and designing novel linear and nonlinear optical components and circuits, as well as for assessing the properties of biological tissues.

\section{MATERIALS AND METHODS}

\section{Quantum WLI}

Figure $1 \mathrm{~b}$ depicts the new experimental schematic dedicated to spectrally resolved quantum WLI (Q-WLI) intended to overcome the above issues. The quantum white-light source is composed of a continuous-wave pump laser and a nonlinear crystal in which energytime entangled photon pairs are generated through spontaneous parametric downconversion ${ }^{24,25}$. This process obeys the conservation of the energy, that is, $\frac{1}{\lambda_{\mathrm{p}}}=\frac{1}{\lambda_{1}}+\frac{1}{\lambda_{2}}$. Here, $\lambda_{\mathrm{p}, 1,2}$ respectively represent the wavelengths in vacuum of the pump laser photons and the individual photons for each generated pair. Another implication of the conservation of the energy is that the degenerate vacuum wavelength of the emission spectrum is $\lambda^{*}=2 \lambda_{\mathrm{p}}$. We send the paired photons to the interferometer; however, as opposed to standard WLI, we now intentionally unbalance it. This provides us with two advantages: first, we avoid single-photon interference, and second, we obtain a means to distinguish events in which the two photons take opposite paths (strongly delayed arrival times at the interferometer's outputs) or the same path (near-zero arrival time difference) ${ }^{24}$. We postselect the latter events by considering only two-photon coincidence detection events in which both the single-photon detector (SPD) and the singlephoton-sensitive spectrometer fire simultaneously. Our goal is now to observe quantum interference between these two-photon contributions, which necessitates that they be coherent and indistinguishable. Coherence is ensured by operating the interferometer at a path-length difference that is shorter than the coherence length of the pump laser $(\sim 100 \mathrm{~m})$ such that the photon pair contributions are in phase ${ }^{26}$. Indistinguishability concerns mainly the temporal envelope of the photon pair wave packet, which is distorted from its original shape by the dispersion-induced temporal walk-off between the individual photons in the SUT. For standard fibres, this means that pathlength differences up to $\sim 10 \mathrm{~m}$ are acceptable ${ }^{27}$.

Thus, provided that the interferometer is operated in these conditions, near-zero arrival time coincidence detection results in a two-photon NOON state:

$$
|\psi\rangle=\frac{|2\rangle_{\mathrm{r}}|0\rangle_{\mathrm{s}}+e^{\mathrm{i} \phi_{N 00 N}}|0\rangle_{\mathrm{r}}|2\rangle_{\mathrm{s}}}{\sqrt{2}}
$$

Here, the ket vectors, indexed by $s$ and $r$, indicate the number of photons in the reference and SUT arms, respectively, and $\phi_{N 00 N}=\phi$ $\left(\lambda_{1}\right)+\phi\left(\lambda_{2}\right)$. We obtain the spectral dependence of $\phi_{\text {NOON }}$ by computing $\phi\left(\lambda_{1}\right)$ and $\phi\left(\lambda_{2}\right)$ according to Equation (1) and respecting the conservation of the energy during the downconversion process:

$$
\left.\phi_{N 00 N} \approx \frac{\mathrm{d}^{2} n}{\mathrm{~d} \lambda^{2}}\right|_{\lambda^{*}} \cdot \frac{\pi L_{\mathrm{s}} \cdot(\Delta \lambda)^{2}}{\frac{\lambda^{*}}{2}+\Delta \lambda}+\phi_{\mathrm{off}}
$$

Here, $\phi_{\text {off }}=\frac{4 \pi\left(n\left(\lambda^{*}\right) L_{\mathrm{s}}-L_{\mathrm{r}}\right)}{\lambda^{*}}$ is an offset term, and $\Delta \lambda=\lambda-\lambda^{*}$. The phase-dependent two-photon coincidence rate $R$ is then $R \propto 1+\cos$ $\left(\phi_{N 00 N}\right)$. In the past, numerous studies have investigated the term $\phi_{\text {off }}$, as it allows measuring optical phase shifts at constant wavelengths with double resolution compared to the standard approach ${ }^{28-30}$.

We access here, for the first time, the wavelength-dependent term in Equation (3) by recording $R$ as a function of $\Delta \lambda$; that is, the twophoton coincidence rate is measured as a function of the pairedphotons' wavelengths.

This leads to several pertinent purely quantum-enabled features. Due to the use of an energy-time entangled two-photon N00N state, the required precision of equilibrating the interferometer is $\sim 10 \mathrm{~m}$ instead of microns to millimetres in standard $\mathrm{WLI}^{11-14}$. This is particularly interesting for improving the ease of use, as no realignment is necessary when changing the SUT; compared to Equation (1), the third-order term $\frac{\mathrm{d}^{3} n}{\mathrm{~d} \lambda^{3}} \lambda^{*}$ in Equation (3) is cancelled owing to energy-time correlations ${ }^{16}$. Furthermore, the wavelength at which chromatic dispersion is measured, $\lambda^{*}$, need not be extracted from the data, as it is exactly twice the wavelength of the continuous-wave pump laser, $\lambda_{\mathrm{p}}$, and can therefore be known with extremely high accuracy. This means that the quantum strategy allows data fitting using exactly one free parameter, namely, $\frac{\mathrm{d}^{2} n}{\mathrm{~d} \lambda^{2}} \lambda^{*}$, which is an essential 
step towards absolute optical-property determination with high precision without systematic errors. Finally, due to the use of a twophoton N00N state, double resolution of $\frac{\mathrm{d}^{2} n}{\mathrm{~d} \lambda^{2}} \lambda^{*}$ is achieved, enabling measurements on shorter samples and components compared to standard WLI, that is, down to the technologically interesting $\mathrm{mm}$ to $\mathrm{cm}$ scale.

\section{Detailed optical setup and data acquisition}

To benchmark standard and quantum approaches, we used a 1-m long SMF28e fibre from Corning as the SUT. We used the same interferometer for all measurements and actively stabilised it using a reference laser and a piezoelectric transducer on one mirror in the reference arm (additional details are provided in the methods section). This ensured that $\phi_{\text {off }}$ remained constant.

For chromatic dispersion measurements using classical WLI, we used a state-of-the-art superluminescent diode. At the output of the interferometer we measured an average spectral intensity of $\sim 125 \mathrm{pW} \mathrm{nm}^{-1}$ from 1450 to $1650 \mathrm{~nm}$. Interferograms were recorded using a spectrometer from Anritsu (model MS9710B, Atsugi-shi, Japan) with $0.1 \mathrm{~s}$ integration time and $0.5 \mathrm{~nm}$ resolution, which are standard parameters for this kind of measurement ${ }^{11,12}$.

For the Q-WLI approach, the light source was a $780.246 \mathrm{~nm}$ laser pumping a type- 0 periodically poled lithium niobate waveguide. We stabilised the laser wavelength against the $F=2 \rightarrow F^{\prime}=2 \times 3$ hyperfine crossover transition in atomic ${ }^{87} \mathrm{Rb}$ such that $\lambda_{\mathrm{p}}$ and $\lambda^{*}$ were known with a precision of the order of $1 \mathrm{fm}$. The quasi-phase matching in the periodically poled lithium niobate waveguide was
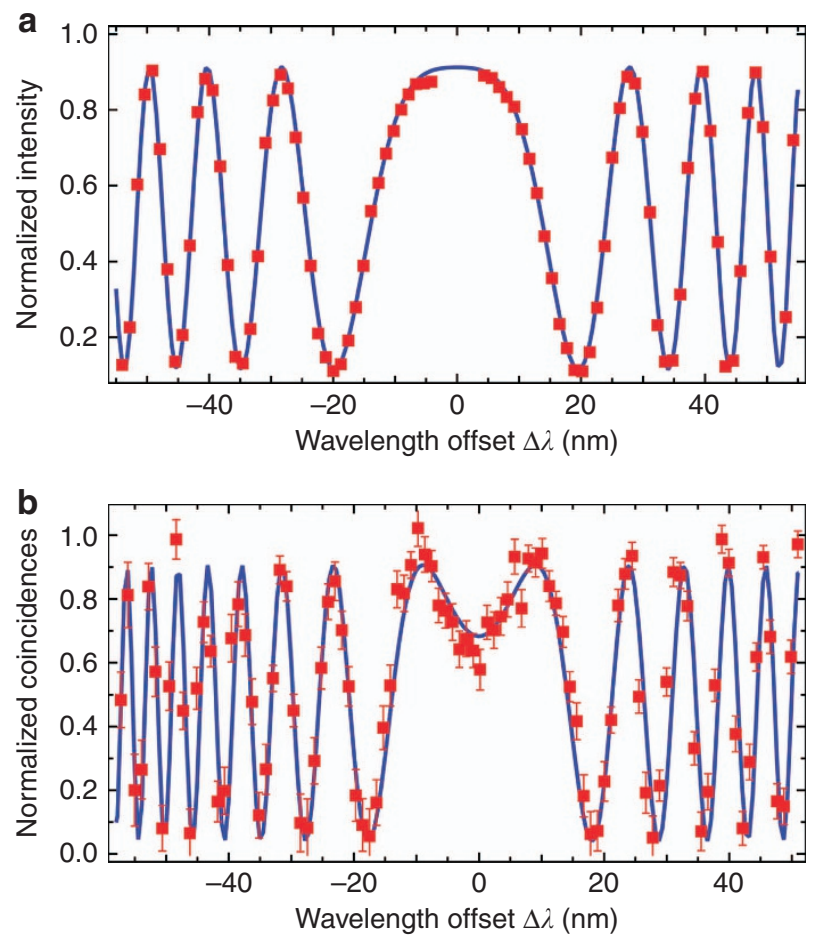

Figure 2 Typical measurements acquired for inferring chromatic dispersion in a 1-m-long standard single-mode fibre. (a) Results obtained with standard WLI, and (b) using Q-WLI. Red dots are data points; blue curves are appropriate fits to the data following Equations (1) and (3), from which $D$ is extracted. Error bars assume Poissonian photon number statistics. For standard WLI, normalization was obtained by measuring two reference spectra. For Q-WLI, normalization was performed on the fly by counting nonzero arrival time difference coincidences. For more details, refer to the Supplementary Information. engineered to generate energy-time entangled photon pairs around the degenerate wavelength of $\lambda^{*}=1560.493 \mathrm{~nm}$ with a bandwidth of $\sim 140 \mathrm{~nm}^{25}$. To detect the paired photons, we used an InGaAs SPD (IDQ 220) at one interferometer output. The single-photon spectrometer at the other output was made of a wavelength-tunable $0.5 \mathrm{~nm}$ bandpass filter followed by another InGaAs SPD (IDQ 230). To avoid saturation of these detectors, the spectral intensity at the interferometer output was reduced to $\sim 25 \mathrm{fW} \mathrm{nm}^{-1}$, which was partially compensated by increasing the integration time to $8 \mathrm{~s}$.

All measurements were repeated 100 times on the same SUT to infer the statistical accuracy of both WLI and Q-WLI approaches.

\section{RESULTS AND DISCUSSION}

Statistical analysis for comparing measurement precision

Typical interference patterns for chromatic dispersion measurements using both methods are shown in Figure $2 \mathrm{a}$ and $2 \mathrm{~b}$. With the Q-WLI setup, we found twice as many interference fringes for the same spectral bandwidth, which is a direct consequence of the doubled phase sensitivity of the two-photon NO0N state. After acquiring $2 \times 100$ measurements on the same SUT, we inferred the precisions of both approaches. The results of the statistical data analysis are shown in Figure 3. For standard WLI, we obtained, on average, $D=$ $17.047 \mathrm{ps}(\mathrm{nm} \mathrm{km})^{-1}$ at $\lambda_{0} \approx 1560.5 \mathrm{~nm}$ with a standard deviation of $\sigma_{\text {classical }}=0.051 \mathrm{ps}(\mathrm{nmkm})^{-1}$. This result is among the most precise reported to date in the literature ${ }^{13,17-22}$. For Q-WLI, we measured, on average, $D=17.035 \mathrm{ps}(\mathrm{nm} \mathrm{km})^{-1}$ at $\lambda^{*}=1560.493 \mathrm{~nm}$ with a significantly smaller standard deviation of $\sigma_{N 00 N}=0.021 \mathrm{ps}(\mathrm{nmkm})^{-1}$.

In our two sets of data, we observed a difference of $0.012 \mathrm{ps}(\mathrm{nm} \mathrm{km})^{-1}$ between the central values, which is larger than the deviation expected from statistical uncertainties $\left(0.006 \mathrm{ps}(\mathrm{nmkm})^{-1}\right)$. Polarization mode dispersion can be excluded as it would introduce at most an offset of $0.003 \mathrm{ps}(\mathrm{nmkm})^{-1}$. Consequently, the difference in central values must originate from systematic errors. In this sense, we compute that, for standard WLI, the difference can be explained by either a slight wavelength offset of the spectrometer $(<0.2 \mathrm{~nm})$ or by a slightly unbalanced interferometer $(\sim 1.5 \mu \mathrm{m})$. Both types of errors induce an error of the fitting parameter $\lambda_{0}$ that translates to an error in $\frac{\mathrm{d}^{2} n}{\mathrm{~d} \lambda^{2}} \mid \lambda_{0}$ (Refs. 11,12). At this point, we emphasise again that in our Q-WLI approach, $\lambda^{*}$ is known with essentially absolute accuracy, and an unbalanced interferometer does not influence the measurement. Because Q-WLI presents fewer sources of systematic errors, it is therefore natural to conclude that Q-WLI determines chromatic dispersion with absolute accuracy.

We further emphasise that our measurements performed with Q-WLI involve $\sim 62$ times fewer photons transmitted through the SUT compared to standard WLI. It is therefore interesting to compare the achievable precision normalised to the number of transmitted photons. For each standard and quantum interferogram, the number of photons reaching the interferometer outputs was $N_{\text {std }} \approx 2.0 \times 10^{10}$ and $N_{\text {quant }} \approx 3.1 \times 10^{8}$, respectively. Consequently, the standard and quantum methods achieve precisions of $(\Delta D)_{\text {std }}=7146 \mathrm{ps}(\mathrm{nm} \mathrm{km})^{-1}$. ${\sqrt{N_{\text {std }}}}^{-1}$ and $(\Delta D)_{\text {quant }}=372 \mathrm{ps}(\mathrm{nm} \mathrm{km})^{-1} \cdot{\sqrt{N_{\text {quant }}}}^{-1}$, respectively. In other words, in addition to being more prone to systematic errors, the standard measurement requires 369 times more photons to achieve the same precision as Q-WLI.

\section{Device calibration using Q-WLI}

Another advantage provided by Q-WLI lies in its straightforward device calibration. All of the optical components in the interferometer actually show small residual chromatic dispersion, and this undesired 


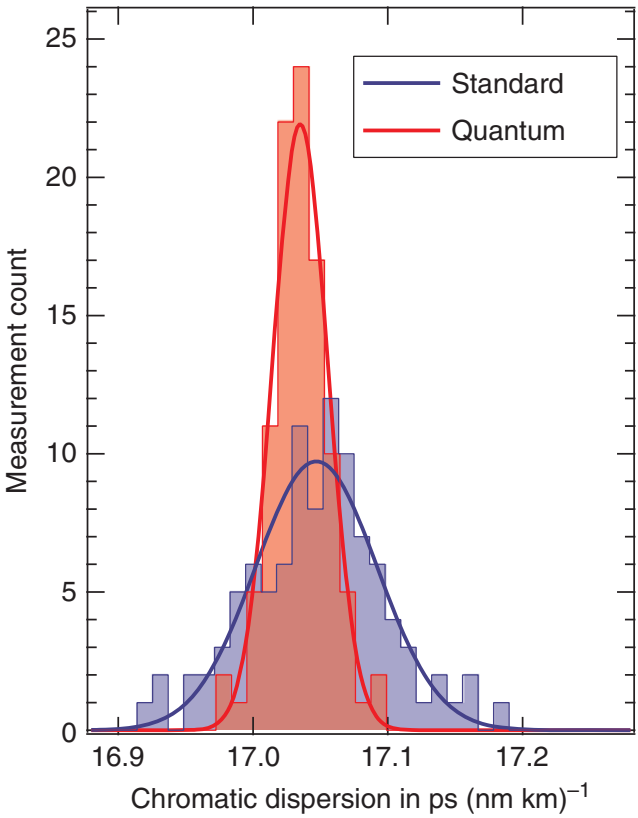

Figure 3 Histogram of inferred chromatic dispersion coefficients after 100 repetitions with the same SUT for both standard (blue) and quantumenhanced (red) measurements. Fits to the data assumed a normal distribution.

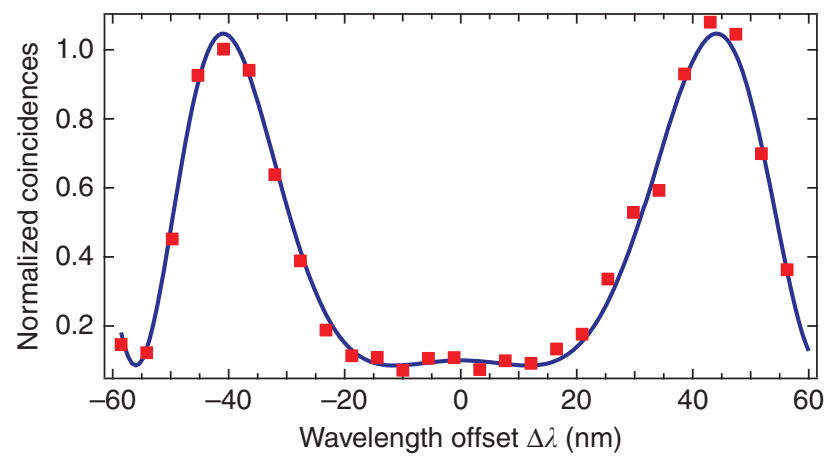

Figure 4 Experimental results when using Q-WLI for inferring residual chromatic dispersion in our interferometer without the SUT. Red dots, data points; the blue curve is an appropriate fit to the data following Equation (3).

offset needs to be evaluated and subtracted from the data to avoid systematic errors. In both cases, this implies performing a measurement without any SUT.

Note that in standard WLI, removing the SUT significantly unbalances the interferometer, and to observe interference, the length of the reference arm must be reduced accordingly (typically on the order of $1 \mathrm{~m}$ ). This procedure is technically challenging, timeconsuming, and might lead to additional systematic errors.

At this point, Q-WLI demonstrates its ability for user-friendly operation. Even after removing the SUT, interference is observed without any interferometer realignment. Figure 4 shows the experimental results that we have obtained when measuring chromatic dispersion in our bare interferometer, that is, without the SUT. It turned out that in our interferometer, residual chromatic dispersion amounted to $\sim 10 \%$ of the measured values on the $1 \mathrm{~m}$ SUT. For all of the data discussed above, except for the raw data in
Figure $2 \mathrm{a}$ and $2 \mathrm{~b}$, we have subtracted the residual chromatic dispersion.

\section{CONCLUSIONS}

We have introduced and demonstrated the concept of spectrally resolved Q-WLI, exploiting energy-time entangled two-photon N00N states. Compared to standard measurements, the N00N state permits achieving a phase sensitivity higher by a factor of two. More strikingly, this use of such quantum states of light reduces the number of free parameters for fitting experimental data from three to one, representing a major advantage for determining optical properties with high precision and absolute accuracy. In addition, our setup does not require a balanced interferometer for performing the measurement, which represents a significant time-saving advantage compared to standard WLI. This is of particular interest for device calibration and when measuring a large set of samples.

As an exemplary demonstration, we have applied our method to infer chromatic dispersion in a standard single-mode fibre, obtaining 2.4 times more precise results compared to state-of-the-art realizations, despite using $\sim 62$ times fewer photons.

We note that the sensitivity of our approach could be further doubled by using a double-pass configuration ${ }^{18}$; this could achieve measurements on short samples, such as optical components and waveguide structures ( $\mathrm{mm}$ to $\mathrm{cm}$ length scale). Such measurements would also be of interest for medical applications where precise knowledge of chromatic dispersion in tissues is required to yield optimal image quality in optical coherence tomography ${ }^{31}$. From this perspective, the reduced number of photons required for quantum WLI is also highly interesting for measurements performed on photosensitive biological samples ${ }^{32-34}$. In optical telecommunication systems, by rotating the polarizations of the entangled photon pairs, our setup could be used for measuring polarization mode dispersion in optical components, which would lead to refinement of manufacturing processes.

In addition, total measurement times could be reduced far below $1 \mathrm{~s}$ by using high-speed superconducting detectors with $\sim 3$ orders of magnitude higher saturation levels compared to the InGaAs SPDs used here $^{35}$. Alternatively, quantum-inspired strategies may also prove to be suitable 36,37

In summary, we believe that combining the fundamental and conceptual advantages enabled by quantum light is a very promising approach for the future development and improvement of applications requiring absolute and high-precision measurements of optical properties.

\section{CONFLICT OF INTEREST}

The authors declare no conflict of interest.

\section{ACKNOWLEDGEMENTS}

We acknowledge financial support from the Foundation Simone \& Cino Del Duca, the European Commission for the FP7-ITN PICQUE project (Grant Agreement No. 608062), l'Agence Nationale de la Recherche (ANR) for the CONNEQT, SPOCQ and SITQOM projects (Grants ANR-EMMA-002-01, ANR-14-CE32-0019 and ANR-15-CE24-0005, respectively) and the iXCore Research Foundation. We also thank MT Pérez Zaballos for assistance with statistical data treatment, as well as M Mitchell, T Debuisschert, A Levenson and P Neumann for fruitful discussions.

1 Giovannetti V, Lloyd S, Maccone L. Quantum-enhanced measurements: beating the standard quantum limit. Science 2004; 306: 1330-1336. 
2 Walther P, Pan J-W, Aspelmeyer M, Ursin R, Gasparoni S et al. De Broglie wavelength of a non-local four-photon state. Nature 2004; 429: 158-161.

3 Mitchell MW, Lundeen JS, Steinberg AM. Super-resolving phase measurements with a multiphoton entangled state. Nature 2004; 429: 161-164.

4 Nagata T, Okamoto R, O'Brien JL, Sasaki K, Takeuchi S. Beating the standard quantum limit with four-entangled photons. Science 2007; 316: 726-729.

5 Higgins BL, Berry DW, Bartlett SD, Wiseman HM, Pryde GJ. Entanglement-free Heisenberg-limited phase estimation. Nature 2007; 450: 393-396.

6 Matthews JCF, Politi A, Stefanov A, O'Brien JL. Manipulation of multiphoton entanglement in waveguide quantum circuits. Nat Photonics 2009; 3: 346-350.

7 Afek I, Ambar O, Silberberg Y. High-NOON states by mixing quantum and classical light. Science 2010; 328: 879-881.

8 Giovannetti V, Lloyd S, Maccone L. Advances in quantum metrology. Nat Photonics 2011; 5: 222-229.

9 Brun-Picard J, Djordjevic S, Leprat D, Schopfer F, Poirier W. Practical quantum realization of the ampere from the elementary charge. Phys Rev X 2016; 6: 041051.

10 Migdall AL, Datla RU, Sergienko A, Orszak JS, Shih YH. Absolute detector quantumefficiency measurements using correlated photons. Metrologia 1995; 32: 479-484.

11 Naganuma K, Mogi K, Yamada H. Group-delay measurement using the Fourier transform of an interferometric cross correlation generated by white light. Opt Lett 1990; 15: 393-395.

12 Diddams S, Diels J-C. Dispersion measurements with white-light interferometry. J Opt Soc Am B 1996; 13: 1120-1129.

13 Grósz T, Kovács AP, Kiss M, Szipöcs R. Measurement of higher order chromatic dispersion in a photonic bandgap fiber: comparative study of spectral interferometric methods. Appl Opt 2014; 53: 1929-1937.

14 Galle MA, Saini SS, Mohammed WS, Qian L. Virtual reference interferometry: theory and experiment. J Opt Soc Am B 2014; 29: 3201-3210.

15 Brendel J, Zbinden H, Gisin N. Measurement of chromatic dispersion in optical fibers using pairs of correlated photons. Opt Commun 1998; 151: 35-39.

16 Nasr MB, Saleh BEA, Sergienko AV, Teich MC. Dispersion-cancelled and dispersionsensitive quantum optical coherence tomography. Opt Express 2004; 12: 1353-1362.

17 Hlubina P, Szpulak M, Ciprian D, Martynkien T, Urbańczyk W. Measurement of the group dispersion of the fundamental mode of holey fiber by white-light spectral interferometry. Opt Express 2007; 15: 11073-11081.

18 Labonté L, Roy P, Pagnoux D, Louradour F, Restoin C et al. Experimental and numerical analysis of the chromatic dispersion dependence upon the actual profile of small core microstructured fibres. J Opt A 2006; 8: 933-938.

19 Kardaś TM, Radzewicz C. Broadband near-infrared fibers dispersion measurement using white-light spectral interferometry. Opt Commun 2009; 282: 4361-4365.

20 Hlubina P, Kadulová M, Mergo P. Chromatic dispersion measurement of holey fibres using a supercontinuum source and a dispersion balanced interferometer. Opt Laser Eng 2013; 51: 421-425.

21 Ye QH, Xu C, Liu X, Knox WH, Yan MF et al. Dispersion measurement of tapered airsilica microstructure fiber by white-light interferometry. Appl Opt 2002; 41: 4467-4470
22 Galle MA, Mohammed WS, Qian L, Smith PWE. Single-arm three-wave interferometer for measuring dispersion of short lengths of fiber. Opt Express 2007; 15 16896-16908.

23 Kovács AP, Osvay K, Bor Z, Szipöcs R. Group-delay measurement on laser mirrors by spectrally resolved white-light interferometry. Opt Lett 1995; 20: 788-790.

24 Kaiser F, Issautier A, Ngah LA, Alibart O, Martin A et al. A versatile source of polarization entangled photons for quantum network applications. Laser Phys Lett 2013; 10: 045202.

25 Alibart O, D'Auria V, De Micheli M, Doutre F, Kaiser F et al. Quantum photonics at telecom wavelengths based on lithium niobate waveguides. J Opt 2016; 18: 104001.

26 Franson JD. Bell inequality for position and time. Phys Rev Lett 1989; 62 : 2205-2208.

27 Vergyris P, Kaiser F, Gouzien E, Sauder G, Lunghi T et al. Fully guided-wave photon pair source for quantum applications. Quantum Sci Technol 2017; 2: 024007.

28 Crespi A, Lobino M, Matthews JCF, Politi A, Neal CR et al. Measuring protein concentration with entangled photons. Appl Phys Lett 2012; 100: 233704.

29 Ono T, Okamoto R, Takeuchi S. An entanglement-enhanced microscope. Nat Commun 2013; 4: 2426.

30 Israel Y, Rosen S, Silberberg Y. Supersensitive polarization microscopy using NOON states of light. Phys Rev Lett 2014; 112: 103604.

31 Drexler W, Morgner U, Kärtner FX, Pitris C, Boppart SA et al. In vivo ultrahigh-resolution optical coherence tomography. Opt Lett 1999; 24: 1221-1223.

32 Frigault MM, Lacoste J, Swift JL, Brown CM. Live-cell microscopy_tips and tools. J Cell Sci 2009; 122: 753-767.

33 Celebrano M, Kukura P, Renn A, Sandoghdar V. Single-molecule imaging by optical absorption. Nat Photonics 2011; 5: 95-98.

34 Piliarik M, Sandoghdar V. Direct optical sensing of single unlabelled proteins and superresolution imaging of their binding sites. Nat Commun 2014; 5: 4495.

35 Zadeh IE, Los JWN, Gourgues RBn, Steinmetz V, Bulgarini G et al. Single-photon detectors combining high efficiency, high detection rates, and ultra-high timing resolution. APL Photonics 2017; 2: 111301.

36 Mazurek MD, Schreiter KM, Prevedel R, Kaltenbaek R, Resch KJ. Dispersion-cancelled biological imaging with quantum-inspired interferometry. Sci Rep 2013; 3: 1582

37 Manceau M, Khalili F, Chekhova M. Improving the phase super-sensitivity of squeezingassisted interferometers by squeeze factor unbalancing. N J Phys 2017; 19: 013014

(c) (i) This work is licensed under a Creative Commons Attribution 4.0 cc) International License. The images or other third party material in this article are included in the article's Creative Commons license, unless indicated otherwise in the credit line; if the material is not included under the Creative Commons license, users will need to obtain permission from the license holder to reproduce the material. To view a copy of this license, visit http://creativecommons.org/licenses/by/4.0/

(C) The Author(s) 2018

Supplementary Information for this article can be found on the Light: Science \& Applications' website (http://www.nature.com/lsa). 